\title{
Filamentary structure and magnetic field orientation in Musca ${ }^{\star}$
}

\author{
N. L. J. Cox ${ }^{1, \star \star}$, D. Arzoumanian ${ }^{2}$, Ph. André ${ }^{3}$, K. L. J. Rygl ${ }^{4}$, T. Prusti ${ }^{4}$, A. Men’shchikov ${ }^{3}$, P. Royer ${ }^{1}$, Á. Kóspál ${ }^{5}$, \\ P. Palmeirim ${ }^{3}$, A. Ribas ${ }^{6}$, V. Könyves ${ }^{3}$, J.-Ph. Bernard ${ }^{7,8}$, N. Schneider ${ }^{9}, 10$, S. Bontemps ${ }^{9}$, B. Merin ${ }^{11}$, R. Vavrek ${ }^{11}$, \\ C. Alves de Oliveira ${ }^{12}$, P. Didelon ${ }^{3}$, G. L. Pilbratt ${ }^{4}$, and C. Waelkens ${ }^{1}$
}

1 Instituut voor Sterrenkunde, KU Leuven, Celestijnenlaan 200D, bus 2401, 3001 Leuven, Belgium e-mail: nick.cox@irap.omp.eu

2 Institut d'Astrophysique Spatiale, CNRS (UMR 8617) Université Paris-Sud 11, Bâtiment 121, Orsay, France

3 Laboratoire AIM Paris, Saclay, CEA/DSM, CNRS, Université Paris Diderot, IRFU, Service d'Astrophysique, Centre d'Études de Saclay, Orme des Merisiers, 91191 Gif-sur-Yvette, France

${ }^{4}$ ESA, Directorate of Science, Scientific Support Office, European Space Research and Technology Centre (ESTEC/SCI-S), Keplerlaan 1, 2201 AZ Noordwijk, The Netherlands

5 Konkoly Observatory, Research Centre for Astronomy and Earth Sciences, Hungarian Academy of Sciences, PO Box 67 , 1525 Budapest, Hungary

${ }^{6}$ Department of Astronomy, Boston University, 725 Commonwealth Avenue, Boston, MA 02215, USA

7 Université de Toulouse, UPS-OMP, IRAP, 31028 Toulouse, France

8 Centre d'Étude Spatiale des Rayonnements, IRAP, 9 Av. colonel Roche, BP 44346, 31028 Toulouse, France

9 OASU/LAB Univ. Bordeaux, CNRS, UMR 5804, 33270 Floirac, France

${ }^{10}$ I. Physik. Institut, University of Cologne, 50937 Cologne, Germany

11 European Space Agency (ESA/ESAC), PO Box 78, 28691 Villanueva de la Cañada, Madrid, Spain

12 European Space Agency (ESA), 3700 San Martin Dr, Baltimore, MD 21218, USA

Received 27 July 2015 / Accepted 26 February 2016

\begin{abstract}
Herschel has shown that filamentary structures are ubiquitous in star-forming regions, in particular in nearby molecular clouds associated with Gould's Belt. High dynamic range far-infrared imaging of the Musca cloud with SPIRE and PACS reveals at least two types of filamentary structures: (1) the main $\sim 10$-pc scale high column-density linear filament; and (2) low column-density striations in close proximity to the main filament. In addition, we find features with intermediate column densities (hair-like strands) that appear physically connected to the main filament. We present an analysis of this filamentary network traced by Herschel and explore its connection with the local magnetic field. We find that both the faint dust emission striations and the plane-of-the-sky (POS) magnetic field are locally oriented close to perpendicular to the high-density main filament (position angle $\sim 25-35^{\circ}$ ). The low-density striations and strands are oriented parallel to the POS magnetic field lines, which are derived previously from optical polarization measurements of background stars and more recently from Planck observations of dust polarized emission. The position angles are $97 \pm 25^{\circ}, 105 \pm 7^{\circ}$, and $105 \pm 5^{\circ}$. From these observations, we propose a scenario in which local interstellar material in this cloud has condensed into a gravitationally-unstable filament (with "supercritical" mass per unit length) that is accreting background matter along field lines through the striations. We also compare the filamentary structure in Musca with what is seen in similar Herschel observations of the Taurus B211/3 filament system and find that there is significantly less substructure in the Musca main filament than in the B211/3 filament. We suggest that the Musca cloud may represent an earlier evolutionary stage in which the main filament has not yet accreted sufficient mass and energy to develop a multiple system of intertwined filamentary components.
\end{abstract}

Key words. ISM: individual objects: Musca - ISM: structure - ISM: clouds - stars: formation - infrared: ISM

\section{Introduction}

The formation and evolution of filamentary structure in molecular clouds is believed to be driven by a combination of three forces: global gravity, magnetic fields, and large-scale turbulence cooling. First results from the Herschel Gould Belt Survey (HGBS) have confirmed the ubiquity of filaments in nearby clouds and suggested that these filaments play a central role in the formation of prestellar cores in low-mass

\footnotetext{
^ Herschel is an ESA space observatory with science instruments provided by European-led Principal Investigator consortia and with important participation from NASA.

$\star \star$ Present address: Université de Toulouse, UPS-OMP, Institut de Recherche en Astrophysique et Planétologie, Toulouse; CNRS, IRAP, 9 Av. colonel Roche, BP 44346, 31028 Toulouse Cedex 4, France.
}

star-forming regions (André et al. 2010, 2014). The Herschel observations revealed a characteristic inner filament width of $\sim 0.1 \mathrm{pc}$, independent of the length or column density of the filaments (Arzoumanian et al. 2011; Palmeirim et al. 2013; Alves de Oliveira et al. 2014; Koch \& Rosolowsky 2015), and close to the sonic scale of turbulence in low-density molecular gas. The origin of this characteristic width is not yet understood well but is possibly connected to the process of filament formation by large-scale turbulent compression of interstellar material (Padoan et al. 2001; Arzoumanian et al. 2011; Federrath 2016). Alternatively, the characteristic width may be set by the dissipation mechanism of magneto-hydrodynamic (MHD) waves due to ion-neutral friction (i.e., ambipolar diffusion) in weakly ionized molecular filaments (Hennebelle 2013; Ntormousi et al. 2016). For dense, self-gravitating filaments, continuous accretion of 


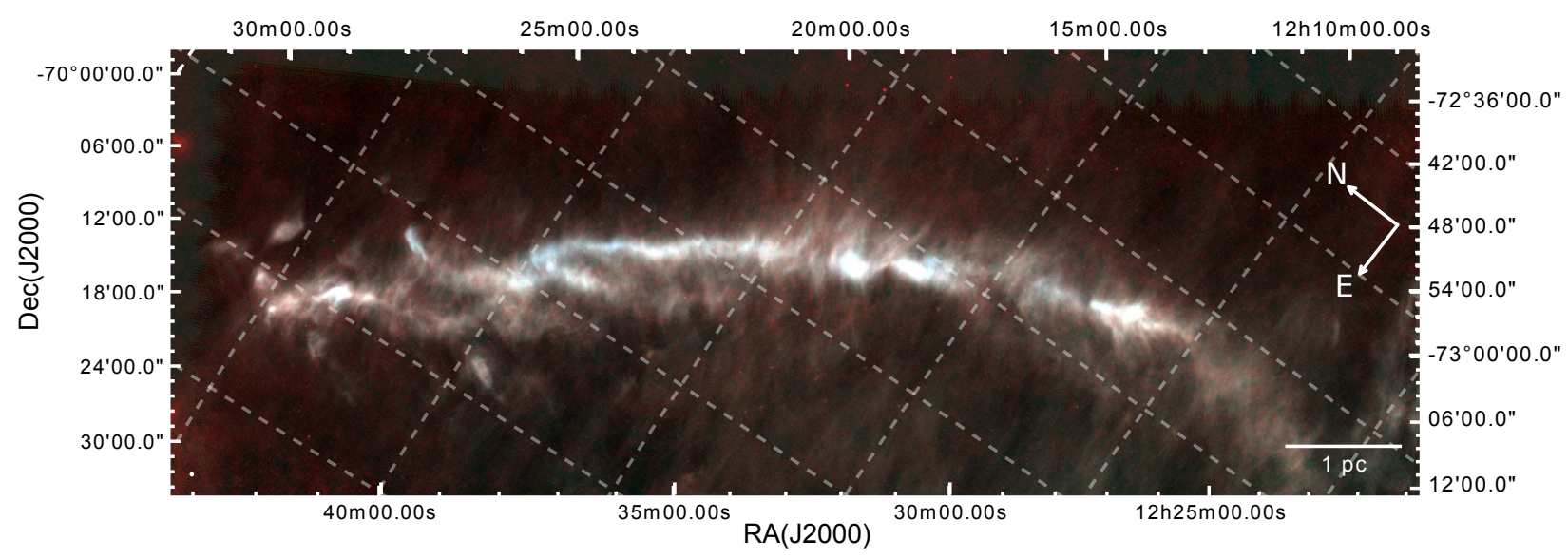

Fig. 1. False colour composite of the Musca molecular cloud. Blue, green, and red correspond to $160 \mu \mathrm{m}, 250 \mu \mathrm{m}$, and $350 \mu \mathrm{m}$ emission, which have beam sizes of $\sim 12^{\prime \prime}, \sim 18^{\prime \prime}$, and $\sim 25^{\prime \prime}$, respectively. The beam size of the $250 \mu \mathrm{m}$ map $\left(\sim 18.2^{\prime \prime}\right)$ is indicated with a white circle in the bottom left corner. The colour intensity scaling is logarithmic. The compass indicates equatorial north and east. North is rotated by $56^{\circ}$ from pointing up. The observed map has a size of about $3^{\circ} \times 1.2^{\circ}$, or $10 \times 5 \mathrm{pc}$ at $200 \mathrm{pc}$.

background cloud material likely plays a key role (Arzoumanian et al. 2013; Heitsch 2013a,b; Hennebelle \& André 2013). Interestingly, filamentary structures are also seen, albeit on different scales, in the molecular material associated with regions of high-mass star- and cluster-formation, such as DR21 (Schneider et al. 2010) and Serpens (Kirk et al. 2013).

The mass per unit length defines whether a filament is unstable to radial gravitational collapse and fragmentation along its length. For an unmagnetized isothermal filament, the critical line mass, $M_{\text {line,crit }}$, is approximately $15-20 M_{\odot} \mathrm{pc}^{-1}$ for typical molecular gas temperatures of 10-12 K (Inutsuka \& Miyama 1997). Herschel observations of nearby clouds are consistent with a simple picture wherein only thermally supercritical filaments $\left(M_{\text {line }}>M_{\text {line,crit }}\right)$ fragment into prestellar cores owing to gravitational instability, subsequently leading to protostellar cores and young stars. Indeed, more than $75 \%$ of the prestellar cores identified with Herschel are located within supercritical filaments, while subcritical filaments $\left(M_{\text {line }}<M_{\text {line,crit }}\right)$ are devoid of such cores (André et al. 2010; Könyves et al. 2015, for example). In addition, molecular line observations of a subset of Herschel filaments show that subcritical filaments have transonic internal velocity dispersions, while supercritical filaments have a velocity dispersion increasing with column density (Arzoumanian et al. 2013).

Observations that relate the orientation of the magnetic field to the elongation of molecular clouds show that they tend to be either parallel or perpendicular to each other (Li et al. 2014). On smaller scales, a comparison between CO maps (Goldsmith et al. 2008), as well as CO velocity anisotropy (Heyer et al. 2008), and the optical polarization revealed an alignment between the local magnetic field and quasi-parallel "striations" of neutral gas in several diffuse low-density subregions of the Taurus molecular cloud. The Herschel dust continuum maps of the Taurus and Chamaeleon clouds taken as part of the HGBS also show the presence of such low-density striations, oriented parallel to the ambient magnetic field and roughly perpendicular to denser, star-forming filaments (Palmeirim et al. 2013; Alves de Oliveira et al. 2014; Koch \& Rosolowsky 2015). At lower resolution, the results of Planck Collaboration Int. XXXII (2016), Planck Collaboration Int. XXXV (2016) indicate that the relative orientation between the plane-of-the-sky (POS) magnetic field and elongated structures in molecular clouds such as filaments changes systematically with column density, where low- and high-column density structures are statistically oriented parallel and perpendicular to the local mean magnetic field, respectively.

The present paper analyses a similar pattern in the case of the Musca cloud. This cloud, which is related to the Chamaeleon low-mass star-forming region, is a $10.5 \mathrm{pc}$ long linear filament (Vilas-Boas et al. 1994) at a distance of 200 pc (in between reported values ranging from 140-225 pc; Franco 1991; Knude \& Hog 1998). The velocity variations observed along the filament are small (a few $\mathrm{km} \mathrm{s}^{-1}$; Vilas-Boas et al. 1994), suggesting that the $3 \mathrm{D}$ orientation of the filament in the cloud may be close to the POS.

While the Musca main filament was known from earlier observations with, e.g., IRAS and NANTEN (Mizuno et al. 2001) and was studied recently in some detail by Kainulainen et al. (2016), the faint striations and hair-like strands observed in the vicinity of the filament (see Fig. 2) are only now revealed thanks to the high spatial and intensity dynamic range of the Herschel data.

In this paper, we present and describe the Herschel HGBS observations of Musca, its filamentary structure and magnetic field orientation (Sect. 2). We tie together the information extracted from Herschel, Planck and near-infrared polarimetry, thus providing a direct one-to-one link between filaments, striations and the magnetic field. In Sect. 3 we discuss our findings in the general context of cloud collapse, and in particular in relation to the filamentary network of the Taurus cloud (Palmeirim et al. 2013).

\section{Far-infrared observations of the Musca cloud}

\subsection{Multi-wavelength imaging and column density and dust temperature maps}

Musca was observed as part of the Herschel Gould Belt Survey (HGBS ${ }^{1}$, André et al. 2010) with the Herschel Space Observatory (Pilbratt et al. 2010) on March 13 and 14, 2011. The PACS (Poglitsch et al. 2010) and SPIRE (Griffin et al. 2010) instruments were used to observe simultaneously at 70, 160, 250, 350 , and $500 \mu \mathrm{m}^{2}$. The observing strategy, data processing, calibration, as well as final map characteristics are similar to the

\footnotetext{
1 http://gouldbelt-herschel. cea.fr

2 The individual PACS and SPIRE maps, as well as the column density and dust temperature maps (at $36.3^{\prime \prime}$ resolution) can be retrieved in FITS
} 

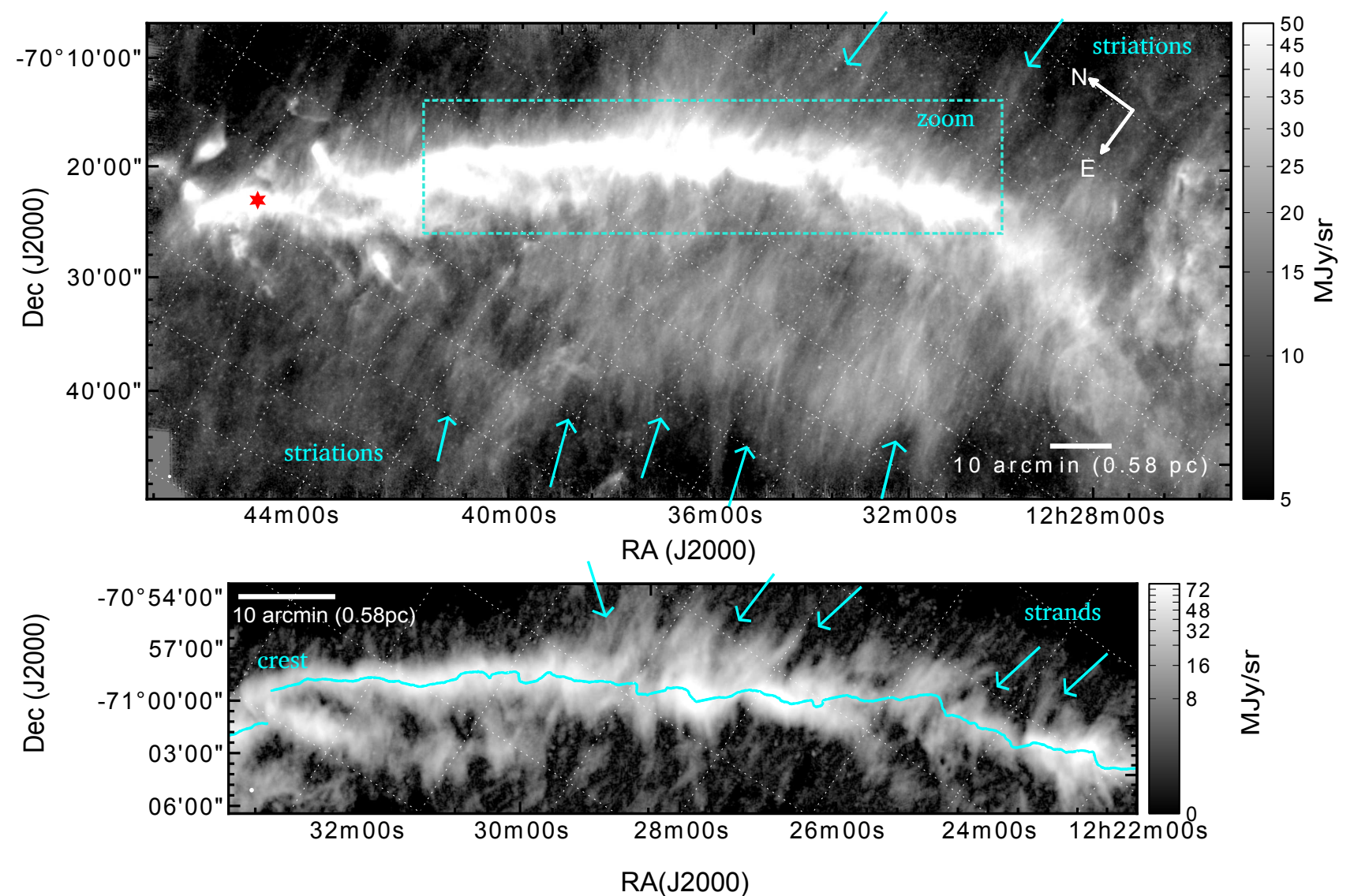

Fig. 2. Two views of the filamentary structure of the Musca cloud showing the striations and strands. Several specific features are indicated with cyan arrows. Top: Herschel/SPIRE $250 \mu \mathrm{m}$ map of the Musca cloud with a "arcsinh" intensity scaling set to enhance visibility of the faint striations. Bottom: curvelet component image of the SPIRE $250 \mu \mathrm{m}$ map ("arcsinh" intensity scaling) produced with the Morphological Component Analysis software from Starck et al. (2003). The crest of the main filament derived with DisPerSE is shown in cyan. This procedure enhances the contrast of the filamentary structure, by removing most non-filamentary background emission. The hair-like strands in the immediate vicinity of the main filament stand out in this close-up view corresponding to the dashed cyan rectangle indicated in the upper panel. The star symbol indicates the position of the young stellar object detected at $70 \mu \mathrm{m}$ (FK5 12:35:16.35, -70:39:56.94 (J2000)). Equatorial north-east directions are indicated by the compass in the upper right.

Chamaeleon maps presented by Spezzi et al. (2013) for example. A false-colour composite far-infrared image of the Musca cloud is shown in Fig. 1.

The molecular hydrogen column density $\left(N_{\mathrm{H}_{2}}\right)$ map was reconstructed at the higher spatial resolution of the SPIRE $250 \mu \mathrm{m}$ map (0.017 pc; Fig. 2) according to the multi-scale decomposition prescription described in Palmeirim et al. (2013). The emission observed from 160 to $500 \mu \mathrm{m}$ was assumed to arise from optically thin thermal radiation from a population of cold dust grains and was approximated by a single-temperature modified blackbody. This scheme also produces a robust dust temperature map at the $36.3^{\prime \prime}$ resolution of the SPIRE $500 \mu \mathrm{m}$ data. The average dust temperature above $A_{V}=2$ mag is $12.9 \mathrm{~K}$.

For consistency with other star-forming regions presented by the HGBS, we adopted the same dust opacity law here $\left(\kappa_{\lambda}=\right.$ $0.1(\lambda / 300 \mu \mathrm{m})^{-\beta} \mathrm{cm}^{2} \mathrm{~g}^{-1}$, assuming a gas-to-dust ratio of 100$)$ as in, e.g., Alves de Oliveira et al. (2014; see Roy et al. 2014 for a discussion on uncertainties). We adopted a mean molecular weight per $\mathrm{H}_{2}$ molecule, $\mu_{\mathrm{H}_{2}}=2.8$, for the $\mathrm{H}_{2}$ surface density map. Both the $\mathrm{H}_{2}$ column density and dust temperature maps are shown in the appendix (Fig. A.1).

format from the Herschel Gould Belt Key Program archive: http:// gouldbelt-herschel.cea.fr/archives
Integrating the $N_{\mathrm{H}_{2}}$ map within the $A_{V}=2 \mathrm{mag}$ contour gives a total mass of $305 M_{\odot}$. This is similar to the total mass of $324 M_{\odot}$ computed directly from the visual extinction map of the Chamaelon-Musca region shown in Schneider et al. (2011) using the canonical conversion factor between $A_{V}$ and $N\left(\mathrm{H}_{2}\right)$ (Bohlin et al. 1978) - as well as using the relation between total mass and $A_{V}$ given by Cambrésy (1999; corrected for the distance adopted here).

\subsection{Filamentary structures}

The Herschel/SPIRE $250 \mu \mathrm{m}$ map shows that the Musca cloud consists of an elongated filament and a network of fainter striations and strands attached and roughly perpendicular to the main structure (Fig. 2). The striations correspond to low-density $\left(<1.8 \times 10^{21} \mathrm{~cm}^{-2}\right.$; see above $)$ filamentary structures observed in close proximity to the main filament, but not necessarily directly connected to it. The striations have a much smaller contrast with respect to the diffuse background emission. Strands are tiny hairlike structures attached to the main filament. The distribution of strands all along the main filament, at a radius $\lesssim 0.5 \mathrm{pc}$ on either side of the central crest (see Fig. 3) suggests that they are an integral part of the main filament structure, and contribute to the power-law tail of the main filament density profile (see Fig. 4b). 


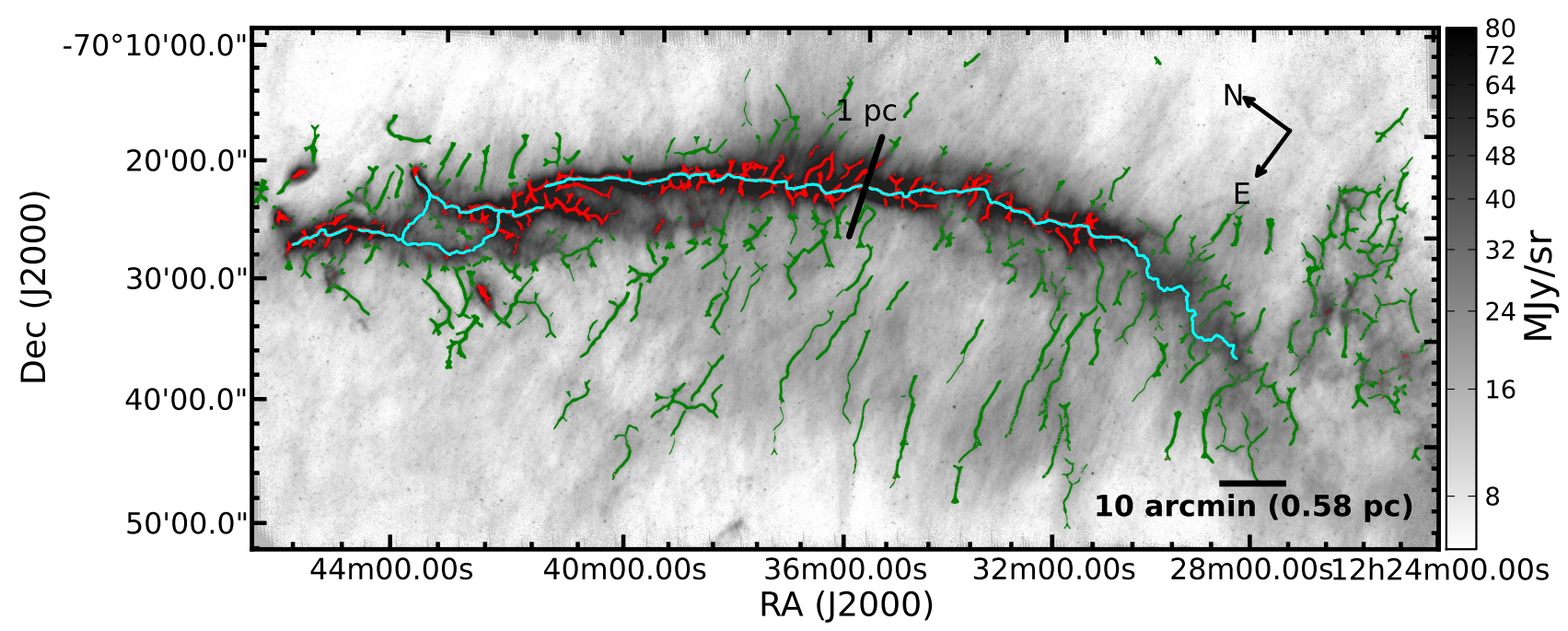

Fig. 3. Herschel/SPIRE $250 \mu \mathrm{m}$ image with the high-density filaments $\left(N\left(\mathrm{H}_{2}\right)>2.7 \times 10^{21} \mathrm{~cm}^{-2}\right)$ overlaid in cyan (crest) and red (strands). Low-density filaments or striations $\left(N\left(\mathrm{H}_{2}\right)<1.8 \times 10^{21} \mathrm{~cm}^{-2}\right)$ as traced by getfilaments are indicated in green (Sect. 2.2). Equatorial north-east directions are indicated by the compass (corresponding to a rotation of $55^{\circ}$ counter-clockwise with respect to north is up). The angular and spatial scales are indicated by the horizontal line in the bottom panel: $10^{\prime}$ is equivalent to $0.58 \mathrm{pc}$.

A detailed view of the filamentary structure (cf. Figs. 3 and 5a) was produced with the multi-scale filament extraction method getfilaments (Men'shchikov 2013). The method identifies and separates filamentary structures from both sources and background by analyzing both the Herschel images and the derived column density map - on a large number of finely-spaced spatial scales ${ }^{3}$. As the appearance and properties of the filamentary component depend on spatial scale, getfilaments reconstructs the intensity distribution of filaments on each scale and determines their single-scale skeletons. The algorithm then accumulates the latter over scales, producing cumulative skeleton images corresponding to the range of spatial scales considered (here, in order to emphasize small-scale filamentary structures, we used angular scales ranging from the SPIRE $250 \mu \mathrm{m}$ beam size of $\sim 18^{\prime \prime}$ up to $72^{\prime \prime}$ or $\sim 0.07 \mathrm{pc}$ at the distance of Musca). Each pixel along such cumulative skeletons contains information on the local significance of the corresponding filament, since the pixel value represents the number of spatial scales at which the filament traced by the skeleton is detectable (cf. Men'shchikov 2013 for details).

For the case of Musca, the maximum getfilaments significance value is 19 for both the filaments and striations. For inclusion in the analysis below we impose a minimum significance value of 2 for both striations and filaments. In the following, and for the purposes of this paper, we make an empirical distinction between high-density and low-density filamentary structures. High-density structures - tracing the main filament - are found to be best identified with $N\left(\mathrm{H}_{2}\right)>2.7 \times 10^{21} \mathrm{~cm}^{-2}$ (maximum at $19.9 \times 10^{21} \mathrm{~cm}^{-2}$ ). Low-density filamentary structures, referred to here as striations, are found to have $N\left(\mathrm{H}_{2}\right)<1.8 \times$ $10^{21} \mathrm{~cm}^{-2}$. Strands are more difficult to separate, but are seen to have column densities between $\approx 2-5 \times 10^{21} \mathrm{~cm}^{-2}$. The pixels associated with the extracted high-density filamentary structures and low-density striations are shown in red and green in Fig. 3. The average column densities for these two types of filamentary structures are $4.2 \times 10^{21}$ and $1.3 \times 10^{21} \mathrm{~cm}^{-2}$ along their crests.

\footnotetext{
3 Here, the smallest angular scale considered was about a third of the SPIRE $250 \mu \mathrm{m}$ beam size or $\sim 6^{\prime \prime}$ and the scaling factor between consecutive scales was 1.073 .
}

In addition, the crest of the main filament was derived with the DisPerSE algorithm (Sousbie 2011) and is shown in Fig. 3 (cyan).

\subsection{Linear mass density}

The column density map was used to constrain the radial density structure of the filament and derive its mass per unit length, $M_{\text {line }}$, as a function of position along the main axis. First, radial column density profiles perpendicular to the main axis (and averaged over an $18^{\prime \prime}$ beam) were constructed at each position along the crest of the filament. Following Arzoumanian et al. (2011) and Palmeirim et al. (2013), we then fitted a Plummer-like function of the form $N_{p}(r)=\frac{1}{\cos i} \times N_{\mathrm{H}_{2}}^{0} /\left[1+\left(r / R_{\mathrm{flat}}\right)^{2}\right]^{\frac{p-1}{2}}$ to the observed radial column density profile $N_{\mathrm{H}_{2}}(r)$ at each position along the filament, where $p$ is the index of the corresponding density profile. We assumed the inclination angle of the filament to the POS to be $i=0^{\circ}$. The average column density profile is best fit by a Plummer function with $R_{\text {flat }}=0.08 \mathrm{pc}$ and $p=2.2 \pm 0.3$ (Fig. $4 \mathrm{~b}$ ). A Gaussian fit to the inner portion of the average column density profile yields a deconvolved FWHM width of $0.14 \pm 0.03$ pc. We note that the values of $R_{\text {flat }}$ and $p$ derived here based on Herschel data agree reasonably well with the values independently found by Kainulainen et al. (2016) using near-infrared extinction data. Local $M_{\text {line }}$ values along the filament were derived by direct integration of the observed profile $N_{\mathrm{H}_{2}}(r)$ over a radius of $0.5 \mathrm{pc}$. An average background corresponding to $N_{\mathrm{H}_{2}} \sim 0.8 \times 10^{21} \mathrm{~cm}^{-2}$ (cf. Fig. 4b) has been subtracted. The direct integration results are consistent with integrating the fitted analytical Plummer profile $N_{p}(r)$ and are shown in Fig. 4a. The mean $M_{\text {line }}$ is $22.3 \pm 7.8 M_{\odot} \mathrm{pc}^{-1}$, which indicates that the Musca filament is marginally thermally supercritical. This derived value of $M_{\text {line }}$ is unlikely to exceed the intrinsic (deprojected) mass per unit length of the filament by more than $15 \%$ since the filament appears to lie close to the POS (see Sect. 1), i.e., $i<30^{\circ}$.

The line-of-sight-averaged dust temperature measured along the crest of the main filament is about $13 \mathrm{~K}$ at the resolution of the $500 \mu \mathrm{m}$ data (Fig. A.1). However, there is a clear negative 

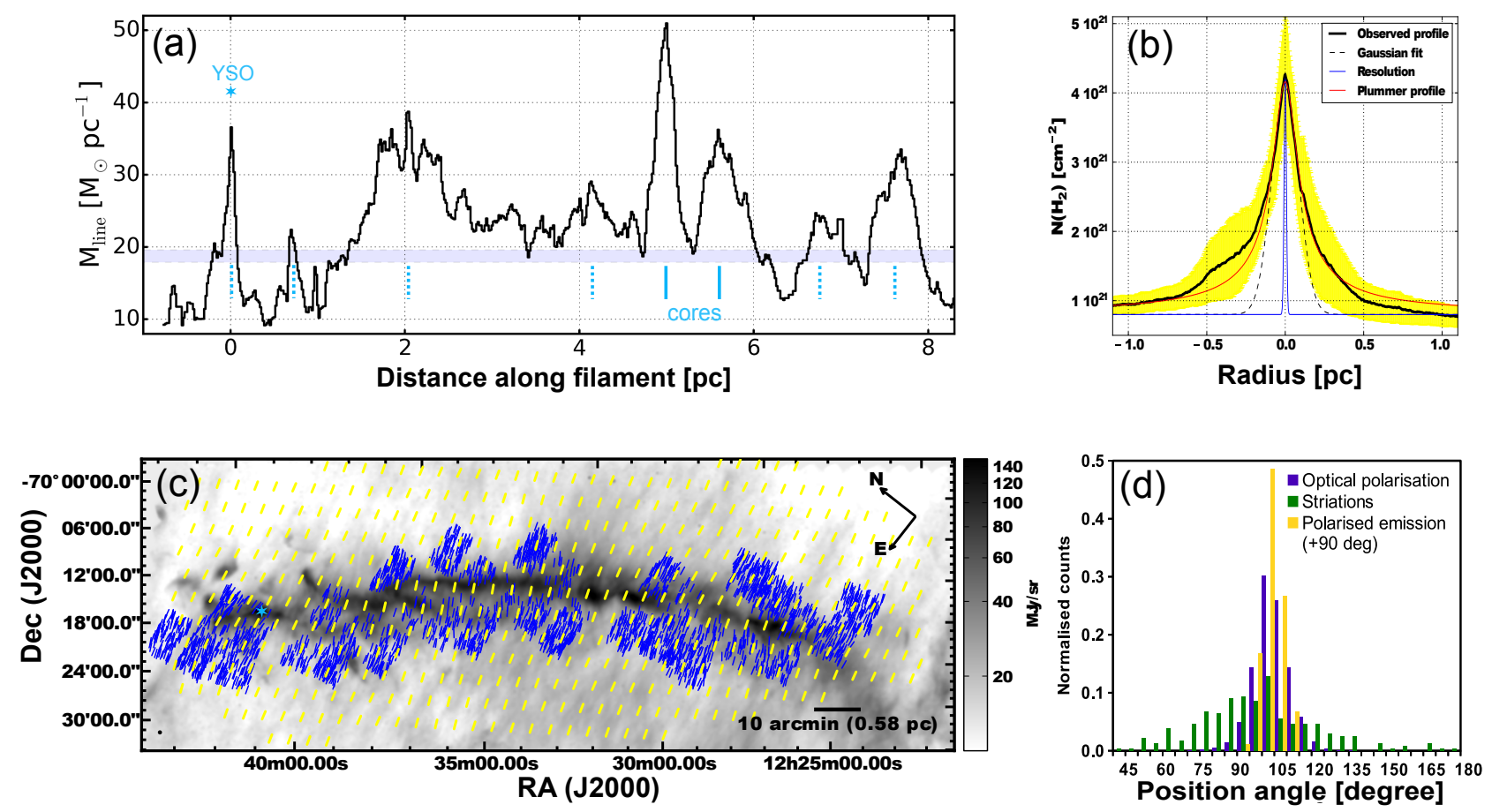

Fig. 4. a) Mass per unit length, $M_{\text {line }}\left(M_{\odot} \mathrm{pc}^{-1}\right)$, along the main filament derived as explained in Sect. 2.3. The light gray area indicates $M_{\text {line,crit }}=17.9-19.6 M_{\odot} \mathrm{pc}^{-1}$. For illustration, the positions of the YSO (Sect. 3) and the cold dense cores studied by Juvela et al. (2010, 2011) are indicated (solid vertical line markers). Several other sharp peaks seen in the $M_{\text {line }}$ graph are related to candidate dense cores (dotted vertical line markers). b) Radial column density profile perpendicular to the major axis of the filament and averaged along its length. The yellow area shows the dispersion along the filament length. The red and dashed curves show the best Gaussian and Plummer function fits (see Sect. 2.3). The inner blue curve corresponds to the $18.2^{\prime \prime}$ beam resolution of the column density maps. c) POS orientations of the magnetic field as derived from starlight polarization (blue lines, Pereyra \& Magalhães 2004) and polarized dust $850 \mu \mathrm{m}$ emission observed by Planck at 9.6' angular resolution (yellow lines of arbitrary length, Planck Collaboration Int. XXXIII 2016) overlaid on the SPIRE $250 \mu \mathrm{m}$ map. Equatorial north-east directions are indicated by the compass (corresponding to a rotation of $55^{\circ}$ counter-clockwise with respect to north is up). The angular and spatial scales are indicated by the horizontal line in the bottom panel: $10^{\prime}$ is equivalent to $0.58 \mathrm{pc}$. d) Distributions of position angles for low-density filaments/striations (green), optical polarization vectors (blue), and polarized emission vectors (rotated by $+90^{\circ}$ to trace the POS magnetic field direction; yellow).

temperature gradient toward the dense inner part of the filament in the map, and the actual dust temperature at the center of the filament is likely lower by more than $\sim 1 \mathrm{~K}$ (cf. Roy et al. 2014). Therefore, we adopt $11-12 \mathrm{~K}$ as the most likely range of dust temperatures at the crest of the main filament and assuming perfect coupling between the gas and the dust temperatures this leads to a range of critical line masses from 17.9-19.6 $M_{\odot} \mathrm{pc}^{-1}$ (indicated by the shaded, light-grey area in Fig. 4a). Note that, for realistic magnetic field strengths (cf. Crutcher 2012), the critical mass per unit length of a magnetized filament is within a factor of order unity from that for an unmagnetized filament (Fiege \& Pudritz 2000).

The variations in $M_{\text {line }}$ along the crest reveal that the main filament is slightly thermally supercritical over most of its length. Nonetheless, there are also a few sections which are slightly subcritical. Sharp peaks in $M_{\text {line }}$ occur at the locations of (candidate) dense cores.

\subsection{Orientations of the magnetic field and filaments}

To study the relative orientation between the POS magnetic field and the filamentary structure in Musca, we derived the distribution of POS magnetic field angles from both optical polarization observations toward field stars (Pereyra \& Magalhães 2004) and polarized dust emission measurements ${ }^{4}$ (Planck Collaboration Int. XXXIII 2016). The resulting position angle distributions are compared to that of the striations in Figs. $4 \mathrm{c}$ and $\mathrm{d}$. The filament position angles (PAs) are measured from Equatorial north to east and correspond to the mean tangential direction along the filaments. The position angle of the main filament ranges from $\mathrm{PA} \sim 25^{\circ}$ to $\mathrm{PA} \sim 35^{\circ}$ along the crest. The mean PA of the striations is $97 \pm 25^{\circ}$. In the area shown in Fig. 3, the mean orientations of the POS magnetic field derived from optical polarization and Planck polarized emission are $105 \pm 7^{\circ}$ and $105 \pm 5^{\circ}$, respectively (see histogram of Fig. 4d). As can be seen in Fig. 4c, the POS magnetic field is highly ordered on parsec scales in the Musca cloud. It is roughly perpendicular to the main filament axis (within $\sim 25^{\circ}$ ) and close to parallel to the striations (within $\sim 10^{\circ}$ ). Interestingly, detailed analysis of the polarization observations presented in Planck Collaboration Int. XXXIII (2016) indicates that the orientation of the POS magnetic field intrinsic to the main filament (derived after subtracting the background contribution to the observed polarized emission) is closer to being perpendicular to the main filament axis by about $15^{\circ}$ (i.e., is within only $\sim 10^{\circ}$ from the normal to the filament axis).

4 Dust polarized emission traces the electric polarization field which, on average, is orthogonal to the mean magnetic field, while optical absorption polarization angles are parallel to the magnetic field lines (Hildebrand et al. 2000; Heiles \& Crutcher 2005). 


\section{Discussion and conclusions}

\subsection{Magnetically-controlled cloud and filament formation}

The observed bimodal distribution of filamentary structures oriented either parallel or perpendicular to the magnetic field direction suggests an "ordered" scenario for the formation of filamentary structures and, subsequently, cores, in a magneto-turbulent interstellar medium. As pointed out by Soler et al. (2013), for example, a large-scale ordered magnetic field can only be maintained if the large-scale turbulence is sub-Alfvénic or at most trans-Alfvénic.

Consider the following sequence. First, large-scale compression of interstellar material due to, for example, an expanding bubble locally creates a finite-sized, self-gravitating layer with a magnetic field primarily in the plane of the dense layer of compressed gas (Inutsuka et al. 2015, for example). Such a dense, self-gravitating layer is expected to fragment into one or several thermally supercritical filaments oriented perpendicular to the mean direction of the magnetic field (cf. Nagai et al. 1998). Additional material from the initial layer will fall onto the newly formed filaments, preferably following the local magnetic field lines (path of least resistance). The anisotropic nature of subAlfvénic turbulence ( $\mathrm{Li}$ et al. 2014, for example) will tend to distribute the accretion flow along low-density, striation-like filaments roughly parallel to the mean local magnetic field. This then gives rise to a bimodal distribution of filamentary structures as observed in Musca. Recent numerical MHD simulations of molecular cloud formation tend to reproduce this pattern (Chen \& Ostriker 2014; Inutsuka et al. 2015, for example). The pattern observed in Musca is reminiscent of the situation seen around the Taurus B211/3 filament system, where velocity information is consistent with accretion of low-density striations onto the denser B211/3 filament (Goldsmith et al. 2008; Palmeirim et al. 2013).

Hennebelle \& André (2013) and Heitsch (2013a,b) independently proposed analytical toy models - based on accretiondriven MHD turbulence - which link the observed accretion phenomenon to the physical reason why supercritical filaments can maintain a roughly constant inner width $\sim 0.1 \mathrm{pc}$ (Arzoumanian et al. 2011) while they should normally contract radially with time (see also the velocity dispersion "evolution" observed by Arzoumanian et al. 2013). Both models emphasize the role of continuing accretion in explaining the observed properties of dense filaments. They differ mainly in the nature of the effective equilibrium at the origin of a nearly constant filament width: balance between accretion-driven MHD turbulence and dissipation of this MHD turbulence by ambipolar diffusion in the model by Hennebelle \& André (2013), balance between turbulence and gravitational fragmentation in the model by Heitsch (2013a,b). The Musca striations and strands provide tantalizing support for continuing accretion of material onto an only marginally thermally supercritical filament, thus supporting this class of models.

Other models predict that a collapsing filament can drag the initially toroidal magnetic field lines along a direction parallel to the filament creating a poloidal field. This poloidal field works to support the cloud radially against self-gravity (Fiege \& Pudritz 2000) but assists collapse and fragmentation along the filament once the critical mass per unit length is reached. If strong enough it will reduce the rate of mass accretion in the radial direction via ambipolar diffusion. Both turbulence and magnetic fields can thus facilitate fragmentation along the filament, and subsequently the formation of prestellar cores. At the resolution of Planck polarization measurements $(0.3 \mathrm{pc}$ at the
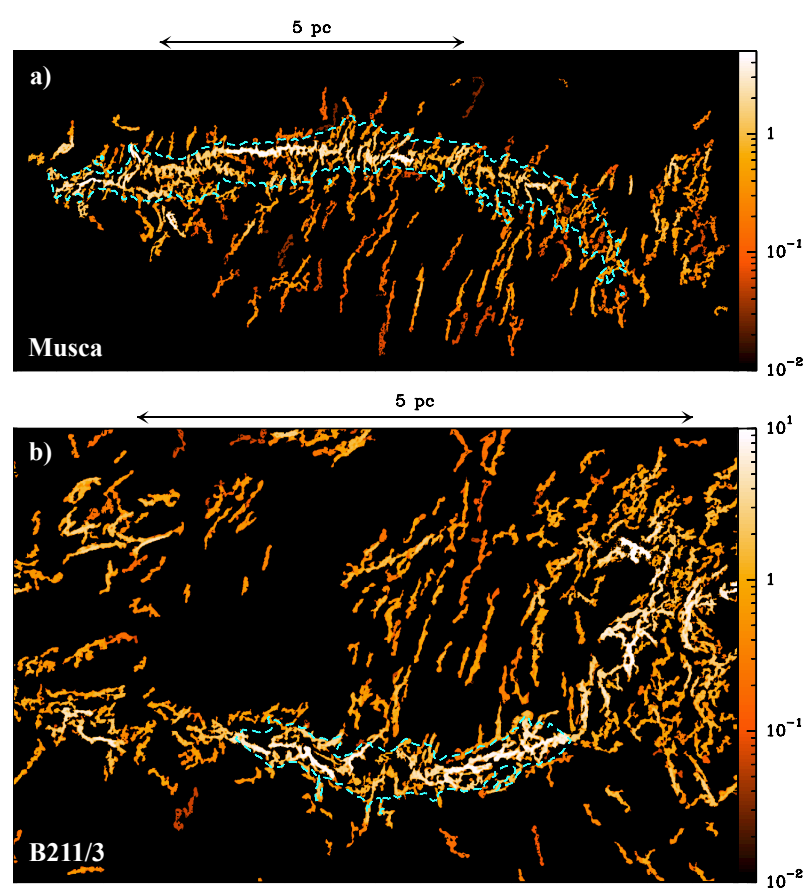

Fig. 5. Filtered versions of the Herschel/SPIRE $250 \mu \mathrm{m}$ images of the Musca a) and Taurus B211/3 b) regions, emphasizing the presence of an organized filamentary network in both cases. The color scales on the right of each panel are in units of $\mathrm{MJy} \mathrm{sr}^{-1}$. Both images were obtained by applying the multi-scale decomposition algorithm getfilaments (Men'shchikov 2013) to the Herschel data, filtering out all transverse angular scales larger than $72^{\prime \prime}$ (i.e., $\sim 0.07 \mathrm{pc}$ at the $200 \mathrm{pc}$ distance of Musca and $\sim 0.05 \mathrm{pc}$ at the $140 \mathrm{pc}$ distance of Taurus). In both cases, the dashed cyan contour outlines the portion of the main filament which was considered for the comparison shown in Fig. 6.

distance of Musca) there is no evidence yet for a poloidal field component within the Musca filament (Planck Collaboration Int. XXXIII 2016). Higher angular resolution polarimetric observations will be required to investigate the magnetic field structure on smaller scales within the filament.

\subsection{Comparison with the Taurus B211/3 filamentary system}

As noted above, the Musca filament bears some resemblance to the well-documented B211/3 filament in Taurus. In both cases, the main filament is $\sim 10 \mathrm{pc}$ long and is surrounded by a series of roughly perpendicular striations which are well-aligned with the ambient magnetic field. One of the main differences is that the mean mass per unit length of the B211/3 filament is more than a factor of 2 higher than that of the Musca filament (i.e., $\sim 50 M_{\odot} \mathrm{pc}^{-1}$ for B211/3 versus $\sim 20 M_{\odot} \mathrm{pc}^{-1}$ for Musca; cf. Palmeirim et al. 2013 and Sect. 2.3). Another difference is that the B211/3 filament is known to exhibit fine substructure in the form of a bundle of intertwined velocity-coherent fibres detected in $\mathrm{C}^{18} \mathrm{O}(1-0)$ and $\mathrm{N}_{2} \mathrm{H}^{+}$(Hacar et al. 2013). These components were also identified in the Herschel/SPIRE $250 \mu$ m data after enhancing the contrast of small-scale structures with getfilaments (see Fig. 2 of André et al. 2014 and Fig. 5b here). While we do not have detailed velocity information in the Musca case, we can use getfilaments to filter out large-scale emission and enhance the contrast of small-scale structures in the Herschel data in the same way as was done for the Taurus B211/3 filamentary system. The result is shown in Fig. 5a and displayed by the red/green skeletons in Fig. 3. Comparison of both panels in Fig. 5 (see also 


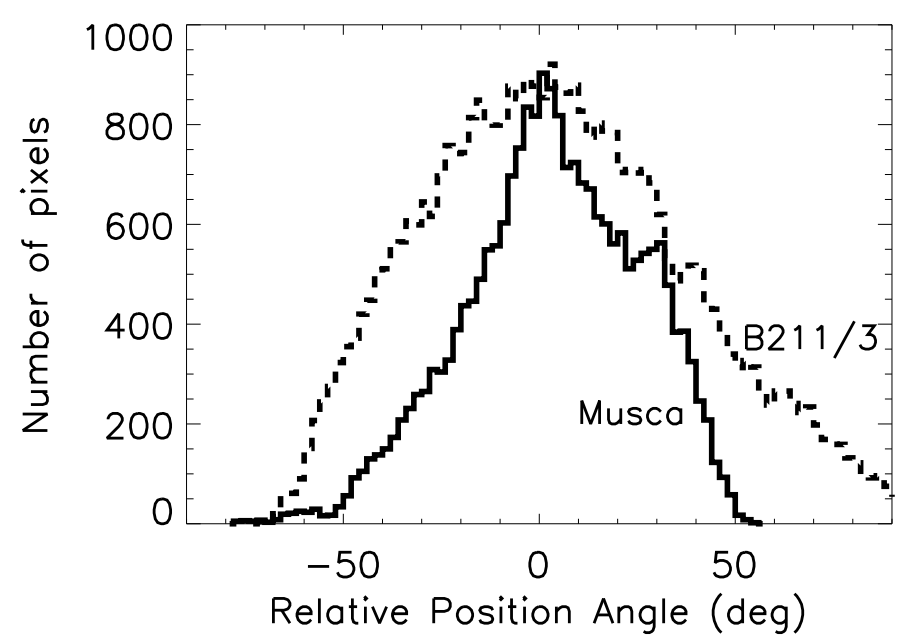

Fig. 6. Distributions of position angles for the local tangential directions to the fine filamentary structures, excluding strands and striations, detected by getfilaments toward the Musca main filament (solid histogram) and the B211/B213 filament (dashed histogram), relative to the mean central crest of the main filament in each case. Note how the distribution of position angles observed in Taurus B211/3 is broader (with a standard deviation of $\sim 35^{\circ}$ ) than the distribution observed in Musca (which has a standard deviation of $\sim 22^{\circ}$ ). This reflects the presence of multiple intertwined fibres in B211/3 (Hacar et al. 2013, and Fig. 5b), while the Musca main filament exhibits significantly less substructure (Fig. 5a).

Fig. 2b of André et al. 2014) suggests that there is significantly less substructure within the Musca filament, and no evidence for intertwined velocity-coherent fibres (cf. Kainulainen et al. 2016; Hacar et al. 2016) as seen for Taurus B211/3.

To quantify this difference further, we compare in Fig. 6 the distribution of position angles for the local tangential directions to the fine filamentary structures - excluding strands and striations (mainly perpendicular to the main filament) - detected by getfilaments in the immediate vicinity of the central crests of the Musca main filament (i.e., area enclosed within the dashed cyan contour in Fig. 3a) and B211/3 main filament (dashed cyan contour in Fig. 3b). It can be seen that this distribution is significantly narrower in Musca (with a standard deviation of $\sim 22^{\circ}$ ) than in Taurus B211/3 (where the standard deviation is $\sim 35^{\circ}$ ).

A possible interpretation of the Taurus B211/3 fibres is that they correspond to the manifestation of accretion-driven MHD turbulence within the main filament structure, resulting from the inflow of background material traced by the magneticallyaligned striations, as described by the model of Hennebelle \& André (2013). Accretion supplies gravitational energy to the system which is then converted into turbulent kinetic energy (cf. Klessen \& Hennebelle 2010) and may explain the increase in velocity dispersion with column density observed by Arzoumanian et al. (2013) for supercritical filaments. In the context of this accretion-driven turbulence picture, we expect a thermally supercritical filament to develop a more complex system of intertwined fibres as the filament grows in mass per unit length and its internal velocity dispersion increases. Since the Musca filament is only marginally supercritical and has only $\sim$ half of the mass per unit length of the Taurus B211/3 filament, it may be representative of a very early stage in the accretion process. Based on an estimated accretion rate of $\sim 27-50 \mathrm{M}_{\odot} \mathrm{pc}^{-1} \mathrm{Myr}^{-1}$, Palmeirim et al. (2013) concluded that the B211/3 filament could roughly double its mass per unit length in $\sim 1-2$ Myr. We speculate that it may take approximately the same time for the Musca filament to acquire a mass per unit length similar to that of the B211/3 filament and develop a multiple system of fibres as observed by Hacar et al. (2013) in the Taurus case. The presence of only one Herschel/PACS $70 \mu \mathrm{m}$ source associated with Musca (IRAS 12322-7023, a candidate young low-mass T Tauri star see Gregorio Hetem et al. 1988) indicates a very low present-day star-formation efficiency of $\ll 1 \%$ and is consistent with the view that the Musca filament is at an earlier stage of evolution compared to the Taurus B211/3 filament. Musca may just be starting to form prestellar cores and protostars while the B211/3 filament has already formed a substantial population of prestellar cores and protostars (Palmeirim et al. 2013; Marsh et al. 2016).

Given its simple pattern and early evolutionary stage, the Musca filament provides an excellent testbed for models of interstellar cylindrical filaments and guide analysis of molecular clouds with more complex filamentary structures. Followup high-resolution molecular line observations (cf. Kainulainen et al. 2016) would be needed to provide detailed kinematical information and assess the validity of our proposed accretion picture.

Acknowledgements. We thank the referee for constructive comments that improved the quality and clarity of this paper. N.L.J.C. and C.A.d.O. kindly acknowledge support from the Faculty of the European Space Astronomy Centre. P.R. and N.L.J.C. acknowledge support from the Belgian Federal Science Policy Office via the PRODEX Programme of ESA. This work was partly supported by the Momentum grant of the MTA CSFK Lenduilet Disk Research Group. This work has received support from the European Research Council under the European Union's Seventh Framework Programme (ERC Advanced Grant Agreements No. 291294 - ORISTARS - and 267934 - MISTIC) and from the French National Research Agency (Grant No. ANR-11-BS56-0010 "STARFICH"). PACS has been developed by a consortium of institutes led by MPE (Germany) and including UVIE (Austria); KUL, CSL, IMEC (Belgium); CEA, OAMP (France); MPIA (Germany); IFSI, OAP/AOT, OAA/CAISMI, LENS, SISSA (Italy); IAC (Spain). This development has been supported by the funding agencies BMVIT (Austria), ESA-PRODEX (Belgium), CEA/CNES (France), DLR (Germany), ASI (Italy), and CICT/MCT (Spain). SPIRE has been developed by a consortium of institutes led by Cardiff Univ. (UK) and including Univ. Lethbridge (Canada); NAOC (China); CEA, LAM (France); IFSI, Univ. Padua (Italy); IAC (Spain); Stockholm Observatory (Sweden); Imperial College London, RAL, UCL-MSSL, UKATC, Univ. Sussex (UK); and Caltech, JPL, NHSC, Univ. Colorado (USA). This development has been supported by national funding agencies: CSA (Canada); NAOC (China); CEA, CNES, CNRS (France); ASI (Italy); MCINN (Spain); SNSB (Sweden); STFC (UK); and NASA (USA).

\section{References}

Alves de Oliveira, C., Schneider, N., Merín, B., et al. 2014, A\&A, 568, A98 André, P., Men'shchikov, A., Bontemps, S., et al. 2010, A\&A, 518, L102 André, P., Di Francesco, J., Ward-Thompson, D., et al. 2014, Protostars and Planets VI, 27

Arzoumanian, D., André, P., Didelon, P., et al. 2011, A\&A, 529, L6

Arzoumanian, D., André, P., Peretto, N., \& Könyves, V. 2013, A\&A, 553, A119

Bohlin, R. C., Savage, B. D., \& Drake, J. F. 1978, ApJ, 224, 132

Cambrésy, L. 1999, A\&A, 345, 965

Chen, C.-Y., \& Ostriker, E. C. 2014, ApJ, 785, 69

Crutcher, R. M. 2012, ARA\&A, 50, 29

Federrath, C. 2016, MNRAS, 457, 375

Fiege, J. D., \& Pudritz, R. E. 2000, MNRAS, 311, 85

Franco, G. A. P. 1991, A\&A, 251, 581

Goldsmith, P. F., Heyer, M., Narayanan, G., et al. 2008, ApJ, 680, 428

Gregorio Hetem, J. C., Sanzovo, G. C., \& Lepine, J. R. D. 1988, A\&AS, 76, 347 Griffin, M. J., Abergel, A., Abreu, A., et al. 2010, A\&A, 518, L3

Hacar, A., Tafalla, M., Kauffmann, J., \& Kovács, A. 2013, A\&A, 554, A55

Hacar, A., Kainulainen, J., Tafalla, M., Beuther, H., \& Alves, J. 2016, A\&A, 584, A97

Heiles, C., \& Crutcher, R. 2005, in Cosmic Magnetic Fields, eds. R. Wielebinski,

\& R. Beck (Berlin: Springer Verlag), Lect. Notes Phys., 664, 137

Heitsch, F. 2013a, ApJ, 769, 115

Heitsch, F. 2013b, ApJ, 776, 62

Hennebelle, P. 2013, A\&A, 556, A153 
Hennebelle, P., \& André, P. 2013, A\&A, 560, A68

Heyer, M., Gong, H., Ostriker, E., \& Brunt, C. 2008, ApJ, 680, 420

Hildebrand, R. H., Davidson, J. A., Dotson, J. L., et al. 2000, PASP, 112, 1215 Inutsuka, S.-I., \& Miyama, S. M. 1997, ApJ, 480, 681

Inutsuka, S.-I., Inoue, T., Iwasaki, K., \& Hosokawa, T. 2015, A\&A, 580, A49

Juvela, M., Ristorcelli, I., Montier, L. A., et al. 2010, A\&A, 518, L93

Juvela, M., Ristorcelli, I., Pelkonen, V.-M., et al. 2011, A\&A, 527, A111

Kainulainen, J., Hacar, A., Alves, J., et al. 2016, A\&A, 586, A27

Kirk, H., Myers, P. C., Bourke, T. L., et al. 2013, ApJ, 766, 115

Klessen, R. S., \& Hennebelle, P. 2010, A\&A, 520, A17

Knude, J., \& Hog, E. 1998, A\&A, 338, 897

Koch, E. W., \& Rosolowsky, E. W. 2015, MNRAS, 452, 3435

Könyves, V., André, P., Men'shchikov, A., et al. 2015, A\&A, 584, A91

Li, H.-B., Goodman, A., Sridharan, T. K., et al. 2014, Protostars and Planets VI, 101

Marsh, K. A., Kirk, J. M., \& André, P. 2016, MNRAS, 459, 342

Men'shchikov, A. 2013, A\&A, 560, A63

Mizuno, A., Yamaguchi, R., Tachihara, K., et al. 2001, PASJ, 53, 1071
Nagai, T., Inutsuka, S.-I., \& Miyama, S. M. 1998, ApJ, 506, 306

Ntormousi, E., Hennebelle, P., André, P., \& Mason, J. 2016, A\&A, 589, A18

Padoan, P., Juvela, M., Goodman, A. A., \& Nordlund, A. 2001, ApJ, 553, 227 Palmeirim, P., André, P., Kirk, J., et al. 2013, A\&A, 550, A38

Pereyra, A., \& Magalhães, A. M. 2004, ApJ, 603, 584

Pilbratt, G. L., Riedinger, J. R., Passvogel, T., et al. 2010, A\&A, 518, L1

Planck Collaboration Int. XXXII. 2016, A\&A, 586, A135

Planck Collaboration Int. XXXIII. 2016, A\&A, 586, A136

Planck Collaboration Int. XXXV. 2016, A\&A, 586, A138

Poglitsch, A., Waelkens, C., Geis, N., et al. 2010, A\&A, 518, L2

Roy, A., André, P., Palmeirim, P., et al. 2014, A\&A, 562, A138

Schneider, N., Csengeri, T., Bontemps, S., et al. 2010, A\&A, 520, A49

Schneider, N., Bontemps, S., Simon, R., et al. 2011, A\&A, 529, A1

Soler, J. D., Hennebelle, P., Martin, P. G., et al. 2013, ApJ, 774, 128 Sousbie, T. 2011, MNRAS, 414, 350

Spezzi, L., Cox, N. L. J., Prusti, T., et al. 2013, A\&A, 555, A71

Starck, J. L., Donoho, D. L., \& Candès, E. J. 2003, A\&A, 398, 785

Vilas-Boas, J. W. S., Myers, P. C., \& Fuller, G. A. 1994, ApJ, 433, 96

\section{Appendix A: Column density and dust temperature maps}
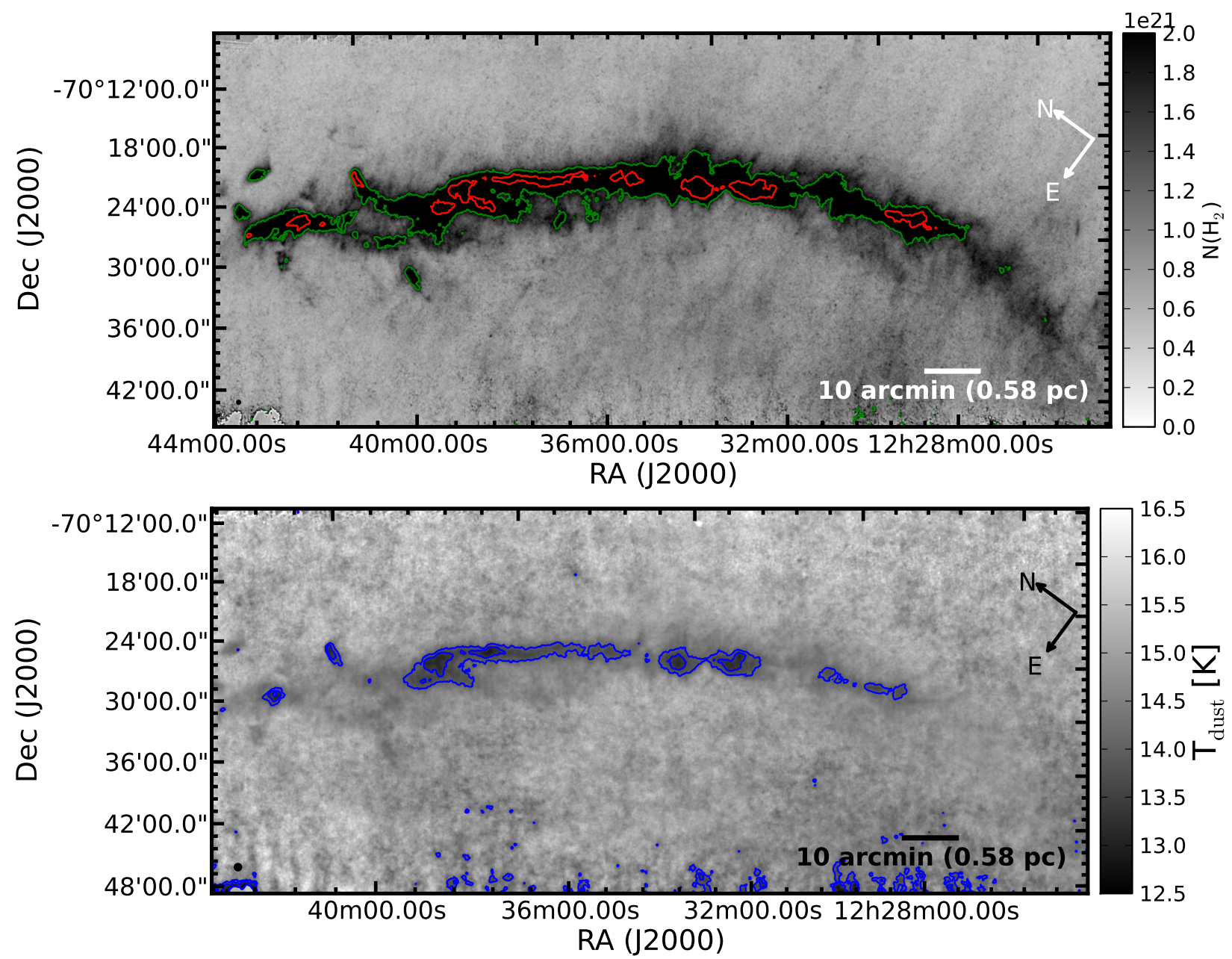

Fig. A.1. Top: $N\left(\mathrm{H}_{2}\right)$ column density map (18.2" resolution) in linear scale (sidebar scale ranges from 0.0 to $2.0 \times 10^{21} \mathrm{~cm}^{-2}$ ). The green contours mark the regions with $N\left(\mathrm{H}_{2}\right) \geq 2.0 \times 10^{21}$. The red contours mark the densest regions of Musca, where $N\left(\mathrm{H}_{2}\right) \geq 4.0 \times 10^{21}$. Bottom: dust temperature map (36.3" resolution) in linear scale (sidebar scale ranges from 12 to $15 \mathrm{~K}$ ). The blue contours correspond to 13.5 and $14.0 \mathrm{~K}$. The compass indicates equatorial north and east. North is rotated by $56^{\circ}$ from up. The observed map has a size of about $3^{\circ} \times 1.2^{\circ}$, or $10 \times 5 \mathrm{pc}$ at $200 \mathrm{pc}$. 